\title{
Sharing Perspectives on Community-Centered Design and International Development
}

\author{
M. Best ${ }^{1}$, A. Dearden ${ }^{2}$, S. Dray ${ }^{3}$, A. Light ${ }^{2}$, J.C. Thomas ${ }^{4}$, Celeste Buckhalter ${ }^{1}$, \\ Daniel Greenblatt ${ }^{1}$, Shanks Krishnan ${ }^{1}$, and Nithya Sambasivan ${ }^{1}$ \\ ${ }^{1}$ Georgia Institute of Technology \\ ${ }^{2}$ Sheffield Hallam University \\ ${ }^{3}$ Dray and Associates \\ ${ }^{4}$ IBM T J Watson Research \\ mikeb@cc.gatech.edu, a.m.dearden@shu.ac.uk, susan.dray@dray.com, \\ annl@dcs.qmul.ac.uk, jcthomas@us.ibm.com
}

\begin{abstract}
Our work with communities in developing countries suggests that HCI practice is a long way from maturity in these contexts. With this SIG, we are seeking to build on a CHI2007 workshop that brought together 50 people to share experience and plan a more integrated approach to the challenges of supporting international development with ICT. We would like to engage a wider cross-section of the community in considering the demands of researching and delivering meaningful design for countries with very different needs from those in the Global North. Our focus will be on issues of development and participation and the impact of differing values in our work.
\end{abstract}

\section{Introduction}

INTERACT 2007 has taken as its theme Socially Responsible Interaction, with an emphasis on universal usability. This SIG, in working to raise awareness of the diversity and impact of cultural and economic conditions around the world, takes universality as its theme and looks at how improving access and participation depends on flexibility and customization in design methods and outcomes. It seeks to gather people interested in how the design of technology can serve communities at different stages of development and with differing value systems, rather than treating them merely as groups of new consumers, or overlooking this unit of social organization.

Researchers and designers will increasingly find themselves on projects that require cross-cultural work in countries labelled as 'developing', as global commercial interests look to open up new markets. However, it is possible to go beyond this interest in other cultures to adopt a more directly engaged approach. This is not to dismiss the importance to individuals of becoming consumers of the new technologies and systems that are currently only available to the industrialized North, especially in situations where access to technology has previously not been a priority or an affordable reality. But a more engaged approach prioritizes working in communities, producing culturally-specific, meaningful, needs-led design for the particular context.

We argue that taking a socially responsible view requires us to go beyond ensuring that technology is usable by everyone, to explore how technologies can emancipate 
and to consider which technologies to prioritize - to community-centered design. This raises a critical question of who makes these decisions and whose values are reflected in implementation.

The organizers of this SIG ran a workshop on the theme of "User-Centered Design and International Development" at CHI 2007 and are now building on the enthusiasm of participants to go beyond discussing means of conducting and employing $\mathrm{HCI}$ research, to consider impact at a community level. We are excited to be collaborating with researchers and practitioners from around the world, including Benin, India, China, Malaysia, Sierra Leone and South Africa, and continuing the development of this network by facilitating this SIG.

\section{Who Should Attend}

This SIG is for two groups of attendees - those with some experience of working in this field who want to compare and share practice, and others with little or no experience, who are interested in learning more. We believe that the ensuing discussions will offer everyone a chance to benefit, by making space to contemplate what UCD means for communities, rather than developers and commercial interests. Since every country has its own developing cultures as well as vulnerable and excluded groups, the approaches we are exploring have something to feed back into mainstream design practice, though we see this primarily as a chance to move the particular challenges of international development onto the agenda. We do not anticipate that all the learning will take place within the SIG, but hope to use it to seed international interest and to channel it into a community of practice(s).

Note: The organisers of this SIG have liaised with those organising the SIG on "Embedding HCI in Developing Countries: localizing content, institutionalizing education and practice". The two SIGs share a common background. But this SIG focuses on development, participation and values. The other SIG focuses upon localization of methods, capacity building, education and institutional support.

\section{Format of Discussions}

After an introduction, we anticipate addressing several issues, with the underlying theme of what constitutes community and development informing our discussions:

1. Whose values should guide us and how are these reflected in our work?

2. What does participation mean in these contexts?

3. How can we learn together, rather than assume that knowledge flows one-way?

4. What should be the agenda to come?

The duration of a SIG will not allow for detailed sharing or debate; therefore, we see this as an important beginning to ongoing dialogue.

We hope to be able to summarize our findings and to integrate them with the findings from the CHI Workshop. In addition, we hope to be able to continue to expand the group of people interested in this area and to develop sustainable networks of linkages for collaboration and support that are truly worldwide. 